\title{
CONVERGENCE ANALYSIS OF FINITE DIFFERENCE METHOD FOR SINGULARLY PERTURBED NONLOCAL DIFFERENTIAL-DIFFERENCE PROBLEM
}

\author{
ERKAN CIMEN AND MUSA CAKIR
}

Received 12 April, 2017

\begin{abstract}
This study is concerned with a singularly perturbed three-point boundary-value problem with delay. Firstly, bounds on the solution and its derivative of the solution to be used later in the paper are obtained. To solve it numerically, we use an exponentially fitted difference scheme on an equidistant mesh which is established by the method of integral identities with the use of exponential basis functions and interpolating quadrature rules with weight and remainder term in integral form. Then, the stability and convergence analysis of difference scheme is given and it is uniformly convergent in perturbation parameter. Furthermore, numerical results which show the effectiveness of the method are presented.
\end{abstract}

2010 Mathematics Subject Classification: 34B10; 34K10; 65L11; 65L12; 65L20

Keywords: singular perturbation, nonlocal condition, finite difference method, initial layer, uniform convergence

\section{INTRODUCTION}

If an ordinary differential equation contains both a perturbation parameter and a delay term, it is called a singularly perturbed differential-difference equation. These equations are a very important role in science and engineering field. For instance, they occur in the study of human pupil light reflex [18], first-exit problems in neurobiology [22], models of physiological processes and diseases [20], optimal control theory [13], optically bistable devices [9] and signal transmission [10], and other models [11].

On the other hand, in recent years many methods have been developed for solving singularly perturbed delay differential problems. In $[8,16]$ some asymptotic analysis of boundary value problems for second order singularly perturbed differentialdifference equations have been considered and some numerical techniques for solving of this type of problems with large and small shifts were considered in [12,17]. Particularly, reproducing kernel method [12], initial value technique [23], some special finite element method [25] have been used for solving these problems. 
Motivated by the above works, we consider the following model problem:

$$
L u:=\varepsilon u^{\prime \prime}(x)+a(x) u^{\prime}(x)+b(x) u(x)+c(x) u(x-r)=f(x), x \in \Omega,
$$

subject to the interval and boundary conditions,

$$
u(x)=\varphi(x), x \in \Omega_{0} ; \gamma u\left(l_{1}\right)+u(l)=A, l_{1} \in \Omega
$$

where $\Omega=\Omega_{1} \cup \Omega_{2}, \Omega_{1}=(0, r], \Omega_{2}=(r, l), \bar{\Omega}=[0, l], \Omega_{0}=[-r, 0]$ and $0<\varepsilon \ll 1$ is the perturbation parameter, $\gamma>0$ and $A$ are given constants. $a(x) \geqslant \alpha>0, b(x) \leqslant$ $0, c(x), f(x)$ and $\varphi(x)$ are given sufficiently smooth functions satisfying certain regularity conditions to be specified and $r$ is a constant delay, which is independent of $\varepsilon$.

The special case $r=0$ and $\varphi(x)=B$ ( $B$ is a real constant) of the above problem so called nonlocal boundary value problem of ordinary differential equation, was initiated by [14]. This kind of problems occur in mathematical models of a large number of phenomena in catalytic processes in chemistry and biology, in problems of semiconductors, in problems of hydromechanics, in studying heat transfer problems and some other physical phenomena [1]. In the survey paper [19], nonlocal problems have been extensively studied many researchers. Nonetheless, the existence of positive solutions for the multipoint boundary value problem with delay is studied in $[5,7,15,24]$. Recently, numerical study of the boundary value problem for linear singularly perturbed differential difference equation with large delay and first type condition is extensively investigated [12,23,25]. But, singularly perturbed nonlocal problems with delay are not studied in literature so far.

It is well known that, for small values of $\varepsilon$, standard numerical methods for solving such problems are unstable and do not give accurate results. For that reason, it is important to develop suitable numerical methods for solving these problems, whose accuracy does not depend on the parameter value $\varepsilon$, i.e., methods that are convergent $\varepsilon$-uniformly. These include fitted finite difference methods, finite element methods using special elements such as exponential elements, and methods which use a priori refined or special non-uniform grids which condense in the boundary layers in a special manner. The various approaches to the design and analysis of appropriate numerical methods for singularly perturbed differential equations can be found in $[2,6,21]$ and the references therein. The numerical method presented here comprises a fitted difference scheme on a uniform mesh. We have derived this approach on the basis of the method of integral identities with the use of interpolating quadrature rules with the weight and remainder terms in integral form. This results in a local truncation error containing only first order derivatives of exact solution and hence facilitates examination of the convergence. The solution of a singularly perturbed problem of the form (1.1)-(1.2) normally has a boundary layer at $x=0$.

This paper is organized as follows. In Section 2, we state some important properties of the exact solution. The description of the finite difference discretization 
have been given in Section 3. In Section 4, we present the error analysis for the approximate solution. Uniform convergence is proved in the discrete maximum norm. In Section 5, we formulate the iterative algorithm for solving the discrete problem and present numerical results which validate the theoretical analysis computationally. The paper ends with a summary of the main conclusions.

Notation. Throughout the paper, $C$ denotes a generic positive constant independent of $\varepsilon$ and the mesh parameter. Some specific, fixed constants of this kind are indicated by subscripting $C$. For any continuous function $g(x)$ denote norms which

$$
\begin{gathered}
\|g\|_{\infty} \equiv\|g\|_{\infty, \bar{\Omega}}=\max _{0 \leq x \leq l}|g(x)|,\|g\|_{1} \equiv\|g\|_{1, \Omega}=\int_{0}^{l}|g(x)| d x, \\
\|g\|_{\infty, k} \equiv\|g\|_{\infty, \bar{\Omega}_{k}}, \quad\|g\|_{1, k} \equiv\|g\|_{1, \Omega_{k}}, \quad k=0,1,2 .
\end{gathered}
$$

\section{Properties of Exact Solution}

Here we give some properties of the solution of (1.1)-(1.2), which are needed in later sections for the analysis of appropriate numerical solution.

Lemma 1. Let $a(x), b(x), c(x), f(x) \in C(\bar{\Omega}), \varphi(x) \in C\left(\Omega_{0}\right)$ and

$$
\lambda:=\alpha^{-1} \beta\left(\|b\|_{1}+\|c\|_{1,2}\right)<1 .
$$

Then for the solution $u(x)$ of the problem (1.1)-(1.2) the following estimates hold:

$$
\begin{gathered}
\|u\|_{\infty} \leqslant C_{0}, \\
\left|u^{\prime}(x)\right| \leqslant C\left(1+\frac{1}{\varepsilon} e^{-\frac{\alpha x}{\varepsilon}}\right), x \in \bar{\Omega},
\end{gathered}
$$

where

$$
\begin{aligned}
C_{0} & =\left[|\varphi(0)|+|A|+\beta\left(|\varphi(0)|+\alpha^{-1}\|f\|_{1}+\alpha^{-1}\|c\|_{\infty, 1}\|\varphi\|_{1,0}\right)\right](1-\lambda)^{-1}, \\
\beta & =1+\gamma l\left(\gamma l_{1}+l\right)^{-1} .
\end{aligned}
$$

Proof. Firstly, we prove (2.2). From (1.1) it follows that

$$
u^{\prime}(x)=u^{\prime}(0) e^{-\frac{1}{\varepsilon} \int_{0}^{x} a(\eta) d \eta}-\frac{1}{\varepsilon} \int_{0}^{x} F(\xi) e^{-\frac{1}{\varepsilon} \int_{\xi}^{x} a(\eta) d \eta} d \xi
$$

with

$$
F(x)=-f(x)+b(x) u(x)+c(x) u(x-r) .
$$

Integrating the relation $(2.4)$ over $(0, x)$ we get

$$
u(x)=\varphi(0)+u^{\prime}(0) \int_{0}^{x} e^{-\frac{1}{\varepsilon} \int_{0}^{\tau} a(\eta) d \eta} d \tau-\frac{1}{\varepsilon} \int_{0}^{x} d \tau \int_{0}^{\tau} F(\xi) e^{-\frac{1}{\varepsilon} \int_{\xi}^{\tau} a(\eta) d \eta} d \xi
$$




$$
=\varphi(0)+u^{\prime}(0) \int_{0}^{x} e^{-\frac{1}{\varepsilon} \int_{0}^{\tau} a(\eta) d \eta} d \tau-\frac{1}{\varepsilon} \int_{0}^{x} d \xi F(\xi) \int_{\xi}^{x} e^{-\frac{1}{\varepsilon} \int_{\xi}^{\tau} a(\eta) d \eta} d \tau .
$$

Using now the condition $\gamma u\left(l_{1}\right)+u(l)=A$, we have

$$
u^{\prime}(0)=\frac{A-(1+\gamma) \varphi(0)+\gamma q\left(l_{1}\right)+q(l)}{\gamma p\left(l_{1}\right)+p(l)}
$$

and so

$$
u(x)=\varphi(0)+\frac{A-(1+\gamma) \varphi(0)+\gamma q\left(l_{1}\right)+q(l)}{\gamma p\left(l_{1}\right)+p(l)} p(x)-q(x),
$$

where

$$
p(x)=\int_{0}^{x} e^{-\frac{1}{\varepsilon} \int_{0}^{\tau} a(\eta) d \eta} d \tau, q(x)=\frac{1}{\varepsilon} \int_{0}^{x} d \xi F(\xi) \int_{\xi}^{x} e^{-\frac{1}{\varepsilon} \int_{\xi}^{\tau} a(\eta) d \eta} d \tau .
$$

In order to prove (2.2), we will use appropriate Green function as an alternative to (2.6).

$$
\begin{aligned}
& L_{*} v:=\varepsilon v^{\prime \prime}(x)+a(x) v^{\prime}(x)=H(x), 0<x<l, \\
& v(x)=\varphi(x),-r \leqslant x \leqslant 0 ; v(l)=A,
\end{aligned}
$$

can be also expressed the solution (2.7) as [3]

$$
v(x)=\left(1-\frac{p(x)}{p(l)}\right) \varphi(0)+\frac{p(x)}{p(l)} A+\int_{0}^{l} G(x, s) H(s) d s
$$

where

$$
\begin{aligned}
G(x, s) & =\frac{1}{\varepsilon} \int_{s}^{l} e^{-\frac{1}{\varepsilon} \int_{s}^{\tau} a(\eta) d \eta} d \tau \times \frac{p(x)}{p(l)}-\frac{1}{\varepsilon} T_{0}(x-s) \int_{s}^{x} e^{-\frac{1}{\varepsilon} \int_{s}^{\tau} a(\eta) d \eta} d \tau, \\
T_{0}(t) & =1, t \geqslant 0 ; T_{0}(t)=0, t<0,
\end{aligned}
$$

and

$$
H(x)=-f(x)+b(x) v(x)+c(x) v(x-r) .
$$

On the other hand, using transformation

$$
u(x)=v(x)-\frac{\gamma x}{\gamma l_{1}+l} v\left(l_{1}\right)
$$

we can rewrite for the solution of the problem (1.1)-(1.2), such that

$$
u(x)=\left(1-\frac{p(x)}{p(l)}\right) \varphi(0)+\frac{p(x)}{p(l)} A+\int_{0}^{l} G(x, s) F(s) d s-\frac{\gamma x}{\gamma l_{1}+l} \int_{0}^{l} G\left(l_{1}, s\right) F(s) d s .
$$


Also in [3] the authors show that $0 \leqslant G(x, s) \leqslant \alpha^{-1}$ for Green's function (2.9), from this point of view

$$
\begin{aligned}
& |u(x)| \leqslant|\varphi(0)|+|A|+\int_{0}^{l}|G(x, s)||F(s)| d s+\frac{\gamma l}{\gamma l_{1}+l} \int_{0}^{l}\left|G\left(l_{1}, s\right)\right||F(s)| d s \\
& \leqslant|\varphi(0)|+|A|+\max _{x, s \in \bar{\Omega}}|G(x, s)| \int_{0}^{l}|F(s)| d s \\
& \quad+\max _{l_{1}, s \in \bar{\Omega}}\left|G\left(l_{1}, s\right)\right| \frac{\gamma l}{\gamma l_{1}+l} \int_{0}^{l}|F(s)| d s \\
& \leqslant|\varphi(0)|+|A|+\alpha^{-1} \beta \int_{0}^{l}|F(s)| d s \\
& \leqslant|\varphi(0)|+|A|+\alpha^{-1} \beta\|f\|_{1}+\alpha^{-1} \beta\|b\|_{1}\|u\|_{\infty} \\
& \quad+\alpha^{-1} \beta \int_{0}^{l}|c(s) u(s-r)| d s .
\end{aligned}
$$

After, by means of changing integral variable by $t=s-r$, we find that

$$
\begin{aligned}
|u(x)| \leqslant & |\varphi(0)|+|A|+\alpha^{-1} \beta\|f\|_{1}+\alpha^{-1} \beta\|b\|_{1}\|u\|_{\infty} \\
& +\alpha^{-1} \beta \int_{-r}^{l-r}|c(r+t) u(t)| d t \\
\leqslant & |\varphi(0)|+|A|+\alpha^{-1} \beta\|f\|_{1}+\alpha^{-1} \beta\|b\|_{1}\|u\|_{\infty} \\
& +\alpha^{-1} \beta \int_{-r}^{0}|c(r+t) \varphi(t)| d t+\alpha^{-1} \beta \int_{0}^{l-r}|c(r+t) u(t)| d t \\
\leqslant & |\varphi(0)|+|A|+\alpha^{-1} \beta\|f\|_{1}+\alpha^{-1} \beta\|b\|_{1}\|u\|_{\infty} \\
& +\alpha^{-1} \beta\|c\|_{\infty, 1}\|\varphi\|_{1,0}+\alpha^{-1} \beta\|u\|_{\infty} \int_{r}^{l}|c(t)| d t \\
\leqslant & |\varphi(0)|+|A|+\alpha^{-1} \beta\|f\|_{1}+\alpha^{-1} \beta\|b\|_{1}\|u\|_{\infty} \\
& +\alpha^{-1} \beta\|c\|_{\infty, 1}\|\varphi\|_{1,0}+\alpha^{-1} \beta\|c\|_{1,2}\|u\|_{\infty}
\end{aligned}
$$


from which (2.2) follows immediately. We have now to prove the estimate (2.3). If we reconsider the terms in (2.5), we obtain the following relations:

$$
\begin{aligned}
\gamma p\left(l_{1}\right)+p(l) & =\gamma \int_{0}^{l_{1}} e^{-\frac{1}{\varepsilon} \int_{0}^{\tau} a(\eta) d \eta} d \tau+\int_{0}^{l} e^{-\frac{1}{\varepsilon} \int_{0}^{\tau} a(\eta) d \eta} d \tau \\
& \geqslant \gamma \int_{0}^{l_{1}} e^{-\frac{a^{*} \tau}{\varepsilon}} d \tau+\int_{0}^{l} e^{-\frac{a^{*} \tau}{\varepsilon}} d \tau=\frac{\varepsilon}{a^{*}}\left[\gamma\left(1-e^{-\frac{a^{*} l_{1}}{\varepsilon}}\right)+1-e^{-\frac{a^{*} l}{\varepsilon}}\right] \\
& \geqslant \frac{\varepsilon}{a^{*}}\left[\gamma\left(1-e^{-a^{*} l_{1}}\right)+1-e^{-a^{*} l}\right] \equiv c_{1} \varepsilon,\left(a^{*}=\|a\|_{\infty}\right),
\end{aligned}
$$

and

$$
\begin{aligned}
\gamma\left|q\left(l_{1}\right)\right|+|q(l)| \leqslant & \frac{\gamma}{\varepsilon} \int_{0}^{l_{1}} d \xi|F(\xi)| \int_{\xi}^{l_{1}} e^{-\frac{1}{\varepsilon} \int_{\xi}^{\tau} a(\eta) d \eta} d \tau \\
& +\frac{1}{\varepsilon} \int_{0}^{l} d \xi|F(\xi)| \int_{\xi}^{l} e^{-\frac{1}{\varepsilon} \int_{\xi}^{\tau} a(\eta) d \eta} d \tau \\
\leqslant & \frac{\gamma}{\varepsilon} \int_{0}^{l_{1}} d \xi|F(\xi)| \int_{\xi}^{l_{1}} e^{-\frac{\alpha(\tau-\xi)}{\varepsilon}} d \tau+\frac{1}{\varepsilon} \int_{0}^{l} d \xi|F(\xi)| \int_{\xi}^{l} e^{-\frac{\alpha(\tau-\xi)}{\varepsilon}} d \tau \\
= & \frac{\gamma}{\varepsilon} \int_{0}^{l_{1}} d \xi|F(\xi)|\left[\alpha^{-1} \varepsilon\left(1-e^{-\frac{\alpha\left(l_{1}-\xi\right)}{\varepsilon}}\right)\right] \\
& +\frac{1}{\varepsilon} \int_{0}^{l} d \xi|F(\xi)|\left[\alpha^{-1} \varepsilon\left(1-e^{-\frac{\alpha(l-\xi)}{\varepsilon}}\right)\right] \\
\leqslant & \alpha^{-1}\left(\gamma \int_{0}^{l_{1}}|F(\xi)| d \xi+\int_{0}^{l}|F(\xi)| d \xi\right) .
\end{aligned}
$$

For $l_{1} \leqslant r$ we get

$$
\gamma\left|q\left(l_{1}\right)\right|+|q(l)| \leqslant \alpha^{-1} \gamma\|f\|_{1}+\alpha^{-1} \gamma\|b\|_{\infty} \int_{0}^{l_{1}}|u(s)| d s
$$




$$
\begin{aligned}
& +\alpha^{-1}(1+\gamma)\|c\|_{\infty} \int_{-r}^{0}|\varphi(s)| d s+\alpha^{-1}\|c\|_{\infty} \int_{0}^{l-r}|u(s)| d s \\
\leqslant & \alpha^{-1} \gamma\left(\|f\|_{1}+\|b\|_{\infty} C_{0} l_{1}\right)+\alpha^{-1}(1+\gamma)\|c\|_{\infty}\|\varphi\|_{1,0} \\
& +\alpha^{-1}\|c\|_{\infty} C_{0}(l-r) \equiv C_{1}
\end{aligned}
$$

and when $l_{1}>r$ we have

$$
\begin{aligned}
\gamma\left|q\left(l_{1}\right)\right|+|q(l)| \leqslant & \alpha^{-1} \gamma\|f\|_{1}+\alpha^{-1} \gamma\|b\|_{\infty} \int_{0}^{l_{1}}|u(s)| d s \\
& +\alpha^{-1}(1+\gamma)\|c\|_{\infty} \int_{-r}^{0}|\varphi(s)| d s \\
& +\alpha^{-1} \gamma\|c\|_{\infty} \int_{0}^{l_{1}-r}|u(s)| d s+\alpha^{-1}\|c\|_{\infty} \int_{0}^{l-r}|u(s)| d s \\
\leqslant & \alpha^{-1} \gamma\left(\|f\|_{1}+\|b\|_{\infty} C_{0} l_{1}\right)+\alpha^{-1}(1+\gamma)\|c\|_{\infty}\|\varphi\|_{1,0} \\
& +\alpha^{-1}\|c\|_{\infty} C_{0}\left(\gamma\left(l_{1}-r\right)+(l-r)\right) \equiv C_{2} .
\end{aligned}
$$

Thus, from (2.5) we are led to

$$
\begin{aligned}
\left|u^{\prime}(0)\right| & \leqslant \frac{|A|+(1+\gamma)|\varphi(0)|+\gamma\left|q\left(l_{1}\right)\right|+|q(l)|}{\gamma p\left(l_{1}\right)+p(l)} \\
& \leqslant \frac{c_{1}^{-1}\left(|A|+(1+\gamma)|\varphi(0)|+\max \left\{C_{1}, C_{2}\right\}\right)}{\varepsilon} \equiv \frac{C_{3}}{\varepsilon} .
\end{aligned}
$$

Using the preceding in (2.4) and arguing as above we arrive at (2.3).

\section{DisCRETE PROBLEM}

Henceforth, we denote by $\omega$ a uniform mesh on $\Omega$ :

$$
\omega=\left\{x_{i}=i h, i=1,2, \ldots, N-1 ; h=l / N\right\}
$$

and $\bar{\omega}=\omega \cup\{x=0, l\}$. For simplicity, we will suppose that $\frac{r}{l} N=N_{0}$ and $\frac{l_{1}}{l} N=N_{1}$ are integers, i.e., $x_{N_{0}}=r$ and $x_{N_{1}}=l_{1}$. Before describing our numerical method, we introduce some notation for the mesh functions. For any mesh function $g(x)$, we use

$$
\begin{gathered}
g_{i}=g\left(x_{i}\right), g_{\bar{x}, i}=\left(g_{i}-g_{i-1}\right) / h, g_{x, i}=\left(g_{i+1}-g_{i}\right) / h, \\
g_{x, i}=\left(g_{x, i}+g_{\bar{x}, i}\right) / 2, g_{\bar{x} x, i}=\left(g_{x, i}-g_{\bar{x}, i}\right) / h,
\end{gathered}
$$




$$
\|g\|_{\infty} \equiv\|g\|_{\infty, \bar{\omega}}=\max _{0 \leqslant i \leqslant N}\left|g_{i}\right|,\|g\|_{1}=\|g\|_{1, \omega}=\sum_{i=1}^{N-1} h\left|g_{i}\right| .
$$

The approach of generating difference method is through the integral identity

$$
h^{-1} \int_{0}^{l} L u(x) \psi_{i}(x) d x=h^{-1} \int_{0}^{l} f(x) \psi_{i}(x) d x, i=1,2, \ldots, N-1
$$

with basis functions

$$
\psi_{i}(x)=\left\{\begin{array}{l}
\psi_{i}^{(1)}(x), x_{i-1}<x<x_{i}, \\
\psi_{i}^{(2)}(x), x_{i}<x<x_{i+1}, \\
0, x \notin\left(x_{i-1}, x_{i+1}\right)
\end{array}\right.
$$

where $\psi_{i}^{(1)}(x)$ and $\psi_{i}^{(2)}(x)$ are the solutions of the following problems, respectively

$$
\begin{aligned}
& \varepsilon \psi^{\prime \prime}(x)-a_{i} \psi^{\prime}(x)=0, \quad x_{i-1}<x<x_{i}, \psi\left(x_{i-1}\right)=0, \quad \psi\left(x_{i}\right)=1, \\
& \varepsilon \psi^{\prime \prime}(x)-a_{i} \psi^{\prime}(x)=0, \quad x_{i}<x<x_{i+1}, \psi\left(x_{i}\right)=1, \quad \psi\left(x_{i+1}\right)=0 .
\end{aligned}
$$

The functions $\psi_{i}^{(1)}(x)$ and $\psi_{i}^{(2)}(x)$ can be explicitly expressed as

$$
\psi_{i}^{(1)}(x)=\frac{e^{a_{i}\left(x-x_{i-1}\right) / \varepsilon}-1}{e^{a_{i} h / \varepsilon}-1}, \psi_{i}^{(2)}(x)=\frac{1-e^{-a_{i}\left(x_{i+1}-x\right) / \varepsilon}}{1-e^{-a_{i} h / \varepsilon}},
$$

which, obviously, satisfy

$$
h^{-1} \int_{x_{i-1}}^{x_{i}+1} \psi_{i}(x) d x=1
$$

Rearranging (3.1), it gives

$$
\begin{aligned}
& -\varepsilon h^{-1} \int_{x_{i-1}}^{x_{i}+1} \psi_{i}^{\prime}(x) u^{\prime}(x) d x+a_{i} h^{-1} \int_{x_{i-1}}^{x_{i}+1} \psi_{i}(x) u^{\prime}(x) d x+b_{i} u_{i}+c_{i} u_{i-N_{0}}=f_{i}-R_{i} \text {, } \\
& i=1,2, \ldots, N-1
\end{aligned}
$$

with

$$
\begin{aligned}
R_{i} & =R_{i}^{(1)}+R_{i}^{(2)}+R_{i}^{(3)}+R_{i}^{(4)}, \\
R_{i}^{(1)} & =h^{-1} \int_{x_{i-1}}^{x_{i+1}}\left[a(x)-a\left(x_{i}\right)\right] u^{\prime}(x) \psi_{i}(x) d x, \\
R_{i}^{(2)} & =h^{-1} \int_{x_{i-1}}^{x_{i+1}}\left[b(x) u(x)-b\left(x_{i}\right) u\left(x_{i}\right)\right] \psi_{i}(x) d x
\end{aligned}
$$




$$
\begin{aligned}
& =h^{-1} \int_{x_{i-1}}^{x_{i+1}} d x \psi_{i}(x) \int_{x_{i-1}}^{x_{i}+1} \frac{d}{d x}(b(x) u(x)) K_{0, i}^{*}(x, \xi) d \xi, \\
R_{i}^{(3)} & =h^{-1} \int_{x_{i-1}}^{x_{i+1}}\left[c(x) u(x-r)-c\left(x_{i}\right) u\left(x_{i}-r\right)\right] \psi_{i}(x) d x \\
& =h^{-1} \int_{x_{i-1}}^{x_{i+1}} d x \psi_{i}(x) \int_{x_{i-1}}^{x_{i+1}} \frac{d}{d x}(c(x) u(x-r)) K_{0, i}^{*}(x, \xi) d \xi, \\
K_{0, i}^{*}(x, \xi) & =T_{0}(x-\xi)-T_{0}\left(x_{i}-\xi\right), 1 \leqslant i \leqslant N-1, \\
R_{i}^{(4)} & =h^{-1} \int_{x_{i-1}}^{x_{i+1}}\left[f\left(x_{i}\right)-f(x)\right] \psi_{i}(x) d x .
\end{aligned}
$$

To be consistent with [3], we present the following approach for exact solution

$$
\ell u_{i} \equiv \varepsilon \theta_{i} u_{\bar{x} x, i}+a_{i} u_{x, i}+b_{i} u_{i}+c_{i} u_{i-N_{0}}=f_{i}-R_{i}, i=1,2, \ldots, N-1 .
$$

where

$$
\theta_{i}=\rho_{i} \operatorname{coth}\left(\rho_{i}\right), \rho_{i}=\frac{a_{i} h}{2 \varepsilon} .
$$

Herefrom, we propose the following difference scheme for approximating (1.1)-(1.2)

$$
\begin{gathered}
\ell y_{i} \equiv \varepsilon \theta_{i} y_{\bar{x} x, i}+a_{i} y_{x, i}+b_{i} y_{i}+c_{i} y_{i-N_{0}}=f_{i}, 0<i<N, \\
y_{i}=\varphi_{i},-N_{0} \leqslant i \leqslant 0 ; \gamma y_{N_{1}}+y_{N}=A,
\end{gathered}
$$

where $\theta$ is given by (3.3).

\section{Stability Bound AND CONVERGENCE}

In this section, we show the convergence of our method. Note that the error function $z_{i}=y_{i}-u_{i}$ is the solution of the discrete problem

$$
\begin{gathered}
\ell z_{i}=R_{i}, 0<i<N, \\
z_{i}=0,-N_{0} \leqslant i \leqslant 0 ; \gamma z_{N_{1}}+z_{N}=0 .
\end{gathered}
$$

where the truncation error $R_{i}$ is given by (3.2).

Lemma 2. If $a(x), b(x), c(x), f(x) \in C^{1}(\bar{\Omega})$ and $\varphi(x) \in C^{1}\left(\Omega_{0}\right)$, then for the truncation error $R_{i}$ we have

$$
\|R\|_{1} \leqslant C h
$$


Proof. Using the mean value theorem, we get

$$
\left|a(x)-a\left(x_{i}\right)\right|=\left|a^{\prime}(\xi)\right|\left|x-x_{i}\right| \leqslant C h, x, \xi \in\left[x_{i-1}, x_{i+1}\right] .
$$

Hence

$$
\left|R_{i}^{(1)}\right| \leqslant C h\left(h^{-1} \int_{x_{i-1}}^{x_{i+1}}\left|u^{\prime}(x)\right| \psi_{i}(x) d x\right)
$$

and taking also into account that $0 \leqslant \psi_{i}(x) \leqslant 1$, we have

$$
\left\|R^{(1)}\right\|_{1} \leqslant C h \sum_{i=1}^{N-1} \int_{x_{i-1}}^{x_{i}+1}\left|u^{\prime}(x)\right| d x \leqslant 2 C h \int_{0}^{l}\left|u^{\prime}(x)\right| d x .
$$

We now use the estimate (2.3) to establish that

$$
\left\|R^{(1)}\right\|_{1} \leqslant C h\left(1+\frac{1}{\varepsilon} \int_{0}^{l} e^{-\frac{\alpha x}{\varepsilon}} d x\right) \leqslant C h\left(1+\alpha^{-1}\left(1-e^{-\frac{\alpha l}{\varepsilon}}\right)\right)=O(h) .
$$

For $R_{i}^{(2)}$, in view of $b \in C^{1}(\bar{\Omega})$ and using Lemma 1, we obtain

$$
\left|R_{i}^{(2)}\right| \leqslant \int_{x_{i-1}}^{x_{i+1}}\left|\frac{d}{d x}[b(x) u(x)]\right|(\xi) d \xi \leqslant C\left(1+\int_{x_{i-1}}^{x_{i}+1}\left|u^{\prime}(\xi)\right| d \xi\right) .
$$

Hence

$$
\left\|R^{(2)}\right\|_{1} \leqslant C h\left(1+\sum_{i=1}^{N-1} \int_{x_{i-1}}^{x_{i}+1}\left|u^{\prime}(\xi)\right| d \xi\right) \leqslant C h\left(1+\int_{0}^{l}\left|u^{\prime}(\xi)\right| d \xi\right) .
$$

Using the (2.3), we get

$$
\left\|R^{(2)}\right\|_{1} \leqslant C h\left(1+\frac{1}{\varepsilon} \int_{0}^{l} e^{-\frac{\alpha \xi}{\varepsilon}} d \xi\right) \leqslant C h\left(1+\alpha^{-1}\left(1-e^{-\frac{\alpha l}{\varepsilon}}\right)\right)=O(h) .
$$

For $R_{i}^{(3)}$, in virtue of $c \in C^{1}(\bar{\Omega})$ and using Lemma 1 , we get

$$
\left|R_{i}^{(3)}\right| \leqslant \int_{x_{i-1}}^{x_{i}+1}\left|\frac{d}{d x}[c(x) u(x-r)]\right|(\xi) d \xi \leqslant C\left(1+\int_{x_{i-1}}^{x_{i}+1}\left|u^{\prime}(\xi-r)\right| d \xi\right) .
$$

Hence

$$
\left\|R^{(3)}\right\|_{1} \leqslant C h\left(1+\sum_{i=1}^{N-1} \int_{x_{i-1}}^{x_{i}+1}\left|u^{\prime}(\xi-r)\right| d \xi\right) \leqslant C h\left(1+\int_{0}^{l}\left|u^{\prime}(\xi-r)\right| d \xi\right)
$$


and, after replacing $s=\xi-r$ this reduces to

$$
\left\|R^{(3)}\right\|_{1} \leqslant C h\left(1+\int_{-r}^{l-r}\left|u^{\prime}(s)\right| d s\right)=C h\left(1+\int_{-r}^{0}\left|\varphi^{\prime}(s)\right| d s+\int_{0}^{l-r}\left|u^{\prime}(s)\right| d s\right)
$$

which yields

$$
\left\|R^{(3)}\right\|_{1} \leqslant C h\left(1+\left\|\varphi^{\prime}\right\|_{1,0}+\alpha^{-1}\left(1-e^{-\frac{\alpha(l-r)}{\varepsilon}}\right)\right)=O(h) .
$$

The same estimate is obtained for $R_{i}^{(4)}$ in the similar manner as above.

Lemma 3. Let $z_{i}$ be the solution (4.1)-(4.2) and (2.1) holds true. Then

$$
\|z\|_{\infty, \omega} \leqslant\|R\|_{1, \omega} .
$$

Proof. We here will use the discrete Green's function $G^{h}\left(x_{i}, s_{j}\right)$ for the operator

$$
\begin{gathered}
L_{*}^{h} \bar{z}_{i}:=-\varepsilon \theta_{i} \bar{z}_{\bar{x} x, i}-a_{i} \bar{z}_{0, i}, \quad 1 \leqslant i \leqslant N-1, \\
\bar{z}_{0}=0, \quad \bar{z}_{N}=0 .
\end{gathered}
$$

As a function of $x_{i}$ for fixed $s_{j}$ this function is being defined as

$$
\begin{gathered}
L_{*}^{h} G^{h}\left(x_{i}, s_{j}\right)=\delta^{h}\left(x_{i}, s_{j}\right), x_{i} \in \omega, s_{j} \in \omega, \\
G^{h}\left(0, s_{j}\right)=G^{h}\left(l, s_{j}\right), s_{j} \in \omega,
\end{gathered}
$$

where $\delta^{h}\left(x_{i}, s_{j}\right)=h^{-1} \delta_{i j}$ and $\delta_{i j}$ is the Kronecker delta. Using Green's function and below transformation

$$
z_{i}=\bar{z}_{i}-\frac{\gamma x_{i}}{\gamma x_{N_{1}}+x_{N}} \bar{z}_{N_{1}}
$$

we can write down the following relation for solution of problem (4.1)-(4.2)

$$
\begin{aligned}
z_{i}= & \sum_{j=1}^{N-1} h G^{h}\left(x_{i}, s_{j}\right)\left(b_{j} z_{j}+c_{j} z_{j-N_{0}}-R_{j}\right) \\
& -\frac{\gamma x_{i}}{\gamma x_{N_{1}}+x_{N}} \sum_{j=1}^{N-1} h G^{h}\left(x_{N_{1}}, s_{j}\right)\left(b_{j} z_{j}+c_{j} z_{j-N_{0}}-R_{j}\right), x_{i} \in \omega .
\end{aligned}
$$

In the analogous manner as in [4] one can show that $0 \leqslant G^{h}\left(x_{i}, s_{j}\right) \leqslant \alpha^{-1}$. Then from (4.5) it follows that

$$
\begin{aligned}
\|z\|_{\infty, \omega} \leqslant & \alpha^{-1}\left\{\|z\|_{\infty, \omega} \sum_{j=1}^{N-1} h\left(\left|b_{j}\right|+\left|c_{j}\right|\right)+\|R\|_{1, \omega}\right\} \\
& +\frac{\gamma x_{N}}{\gamma x_{N_{1}}+x_{N}} \alpha^{-1}\left\{\|z\|_{\infty, \omega} \sum_{j=1}^{N-1} h\left(\left|b_{j}\right|+\left|c_{j}\right|\right)+\|R\|_{1, \omega}\right\}
\end{aligned}
$$




$$
\begin{aligned}
& \leqslant \alpha^{-1} \beta\left\{\|z\|_{\infty, \omega}\left(\|b\|_{1, \omega}+\|c\|_{1, \omega}\right)+\|R\|_{1, \omega}\right\} \\
& \leqslant \lambda\|z\|_{\infty, \omega}+\alpha^{-1} \beta\|R\|_{1, \omega},
\end{aligned}
$$

which implies the validity of (4.4).

Now, we give the main result on $\varepsilon$-uniform convergence of the proposed method for solving the problem (1.1)-(1.2).

Theorem 1. Under the condition of Lemma 1, the solution of (1.1)-(1.2) and the solution (3.4)-(3.5) satisfy the estimate

$$
\|y-u\|_{\infty, \bar{\omega}} \leqslant C h .
$$

Proof. Combining the previous lemmas, we immediately have (4.6).

\section{AlgORITHM AND NUMERICAL RESULTS}

In this section, we shall propose the following iterative technique for solving problem (3.4)-(3.5). In addition, we demonstrate the effectiveness of the present method by applying it to three examples of problem (1.1)-(1.2).

$$
\begin{gathered}
\varepsilon \theta_{i} y_{\bar{x} x, i}^{(n)}+a_{i} y_{0, i}^{(n)}+b_{i} y_{i}^{(n)}=f_{i}^{(1)}, f_{i}^{(1)}=f_{i}-c_{i} y_{i-N_{0}}, 1 \leq i \leq N_{0}-1, \\
y_{i}^{(n)}=\varphi_{i},-N_{0} \leq i \leq 0 ; y_{N_{0}}^{(n)}=\kappa_{n-1}, \\
\varepsilon \theta_{i} y_{\bar{x} x, i}^{(n)}+a_{i} y_{0}^{(n)}+b_{i} y_{i}^{(n)}=f_{i}^{(2)}, f_{i}^{(2)}=f_{i}-c_{i} y_{i-N_{0}}^{(n)}, N_{0}+1 \leq i \leq N-1, \\
y_{N_{0}}^{(n)}=\kappa_{n-1}, y_{N}^{(n)}=A-\gamma \mu_{n-1},
\end{gathered}
$$

where $\kappa_{n}$ and $\mu_{n}$ are values of (3.4) for $y_{N_{0}}$ and $y_{N_{1}}$ so that

$$
\begin{gathered}
\kappa_{n}=\left[\varepsilon \theta_{N_{0}}\left(y_{N_{0}+1}^{(n)}+y_{N_{0}-1}^{(n)}\right)+h^{2}\left(a_{N_{0}} y_{0, N_{0}}^{(n)}+c_{N_{0}} \varphi_{0}-f_{N_{0}}\right)\right]\left(2 \varepsilon \theta_{N_{0}}-b_{N_{0}} h^{2}\right)^{-1}, \\
\mu_{n}=\left\{\begin{array}{cl}
\frac{\varepsilon \theta_{N_{1}}\left(y_{N_{1}+1}^{(n)}+y_{N_{1}-1}^{(n)}\right)+h^{2}\left(a_{N_{1}} y_{0}^{(n)}+c_{N_{1}} \varphi_{N_{1}-N_{0}}-f_{N_{1}}\right)}{2 \varepsilon \theta_{N_{1}}-b_{N_{1}} h^{2}}, & N_{0} \geq N_{1}, \\
\frac{\varepsilon \theta_{N_{1}}\left(y_{N_{1}+1}^{(n)}+y_{N_{1}-1}^{(n)}\right)+h^{2}\left(a_{N_{1}} y_{0}^{(n)}+c_{N_{1}} y_{N_{1}-N_{0}}^{(n)}-f_{N_{1}}\right)}{2 \varepsilon \theta_{N_{1}}-b_{N_{1}} h^{2}}, & N_{0}<N_{1}, \\
n=1,2,3, \ldots, &
\end{array}\right.
\end{gathered}
$$

$\kappa_{0}=\mu_{0}=C_{0}$, and $C_{0}$ is given by (2.2).

We consider the following test problems:

Example 1. (Case 1: $l_{1}<r$ )

$$
\left\{\begin{array}{l}
\varepsilon u^{\prime \prime}(x)+64 u^{\prime}(x)-u(x)+0.25 u(x-1)=0.25 x-1.25,0<x<\frac{3}{2} \\
u(x)=x,-1 \leqslant x \leqslant 0 ; \frac{1}{4} u\left(\frac{1}{2}\right)+u\left(\frac{3}{2}\right)=2,
\end{array}\right.
$$


the exact solution is given by

$$
u(x)= \begin{cases}1+d_{1} e^{m_{1} x}+d_{2} e^{m_{2} x}, & x \in[0,1] \\ d_{3} e^{m_{1}(x-1)}+d_{4} e^{m_{2}(x-1)}-\frac{59}{4} & \\ -\frac{1}{4 \sqrt{d}}(x-1)\left(\sqrt{d}+d_{1} e^{m_{1}(x-1)}-d_{2} e^{m_{2}(x-1)}\right), & x \in\left(1, \frac{3}{2}\right]\end{cases}
$$

where

$$
\begin{aligned}
& d_{1}=\frac{\frac{133}{8}+\beta_{1} e^{\frac{m_{1}}{2}}-\left(\beta_{2}-e^{m_{2}}-\frac{1}{8 \sqrt{d}}-\frac{1}{4}\right) e^{\frac{m_{2}}{2}}}{\sigma_{3}-\sigma_{4}+\frac{1}{4}\left(e^{\frac{m_{1}}{2}}-e^{\frac{m_{2}}{2}}\right)} \\
& d_{2}=-1-d_{1}, d_{3}=\alpha_{1} d_{1}-\beta_{1}, d_{4}=-\alpha_{2} d_{1}+\beta_{2}-e^{m_{2}} .
\end{aligned}
$$

Example 2. (Case 2: $l_{1}=r$ )

$$
\left\{\begin{array}{l}
\varepsilon u^{\prime \prime}(x)+64 u^{\prime}(x)-u(x)+0.25 u(x-1)=0.25 x-1.25,0<x<\frac{3}{2} \\
u(x)=x,-1 \leqslant x \leqslant 0 ; \frac{1}{2} u(1)+u\left(\frac{3}{2}\right)=2,
\end{array}\right.
$$

the exact solution is given by

$$
u(x)= \begin{cases}1+d_{1} e^{m_{1} x}+d_{2} e^{m_{2} x}, & x \in[0,1] \\ d_{3} e^{m_{1}(x-1)}+d_{4} e^{m_{2}(x-1)}-\frac{59}{4} & \\ -\frac{1}{4 \sqrt{d}}(x-1)\left(\sqrt{d}+d_{1} e^{m_{1}(x-1)}-d_{2} e^{m_{2}(x-1)}\right), & x \in\left(1, \frac{3}{2}\right]\end{cases}
$$

where

$$
\begin{aligned}
& d_{1}=\frac{\frac{135}{8}+\beta_{1} e^{\frac{m_{1}}{2}}-\frac{1}{2}\left(1-e^{m_{2}}\right)-\left(\beta_{2}-e^{m_{2}}-\frac{1}{8 \sqrt{d}}\right) e^{\frac{m_{2}}{2}}}{\frac{1}{2}\left(e^{m_{1}}-e^{m_{2}}\right)+\sigma_{3}-\sigma_{4}}, \\
& d_{2}=-1-d_{1}, d_{3}=\alpha_{1} d_{1}-\beta_{1}, d_{4}=-\alpha_{2} d_{1}+\beta_{2}-e^{m_{2}} .
\end{aligned}
$$

Example 3. (Case 3: $l_{1}>r$ )

$$
\left\{\begin{array}{l}
\varepsilon u^{\prime \prime}(x)+64 u^{\prime}(x)-u(x)+0.25 u(x-1)=0.25 x-1.25,0<x<\frac{3}{2} \\
u(x)=x,-1 \leqslant x \leqslant 0 ; \frac{1}{2} u\left(\frac{5}{4}\right)+u\left(\frac{3}{2}\right)=2,
\end{array}\right.
$$

the exact solution is given by

$$
u(x)= \begin{cases}1+d_{1} e^{m_{1} x}+d_{2} e^{m_{2} x}, & x \in[0,1] \\ d_{3} e^{m_{1}(x-1)}+d_{4} e^{m_{2}(x-1)}-\frac{59}{4} & \\ -\frac{1}{4 \sqrt{d}}(x-1)\left(\sqrt{d}+d_{1} e^{m_{1}(x-1)}-d_{2} e^{m_{2}(x-1)}\right), & x \in\left(1, \frac{3}{2}\right]\end{cases}
$$

where

$$
\begin{aligned}
d_{1}= & \frac{\frac{777}{32}+\beta_{1} e^{\frac{m_{1}}{4}}\left(\frac{1}{2}+e^{\frac{m_{1}}{4}}\right)-\left(\beta_{2}-e^{m_{2}}-\frac{1}{8 \sqrt{d}}\right) e^{\frac{m_{2}}{2}}}{\sigma_{1}-\sigma_{2}+\sigma_{3}-\sigma_{4}} \\
& -\frac{\left(\frac{\beta_{2}}{2}-\frac{e^{m_{2}}}{2}-\frac{1}{32 \sqrt{d}}\right) e^{\frac{m_{2}}{4}}}{\sigma_{1}-\sigma_{2}+\sigma_{3}-\sigma_{4}},
\end{aligned}
$$




$$
d_{2}=-1-d_{1}, d_{3}=\alpha_{1} d_{1}-\beta_{1}, d_{4}=-\alpha_{2} d_{1}+\beta_{2}-e^{m_{2}},
$$

and the other coefficients for all examples are as follows

$$
\begin{gathered}
\sigma_{k}=\left\{\begin{array}{cc}
\left(\frac{\alpha_{k}}{2}+(-1)^{k} \frac{1}{32 \sqrt{d}}\right) e^{\frac{m_{k}}{4}}, & k=1,2, \\
\left(\alpha_{k-2}+(-1)^{k} \frac{1}{8 \sqrt{d}}\right) e^{\frac{m_{k-2}}{2}}, & k=3,4,
\end{array}\right. \\
\alpha_{1,2}=e^{m_{1,2}+\frac{\varepsilon}{2 d}, \beta_{1,2}=\frac{\varepsilon}{4 \sqrt{d}}\left(63 m_{2,1}-\frac{1}{\sqrt{d}}-1\right),} \\
m_{1}=\frac{-64+\sqrt{d}}{2 \varepsilon}, m_{2}=\frac{-64-\sqrt{d}}{2 \varepsilon}, d=2^{12}+4 \varepsilon .
\end{gathered}
$$

TABLE 1. Errors and convergence rates for Example 1

\begin{tabular}{llllll}
\hline$\varepsilon$ & $N_{0}=64$ & $N_{0}=128$ & $N_{0}=256$ & $N_{0}=512$ & $N_{0}=1024$ \\
\hline $2^{-2}$ & $3.53104 \mathrm{E}-5$ & $1.02837 \mathrm{E}-5$ & $2.69286 \mathrm{E}-6$ & $6.83444 \mathrm{E}-7$ & $3.60809 \mathrm{E}-7$ \\
& 1.78 & 1.93 & 1.98 & 1.92 & \\
$2^{-4}$ & $5.75563 \mathrm{E}-5$ & $2.46914 \mathrm{E}-5$ & $8.83647 \mathrm{E}-6$ & $2.57391 \mathrm{E}-6$ & $6.74033 \mathrm{E}-7$ \\
& 1.22 & 1.48 & 1.78 & 1.93 & \\
$2^{-6}$ & $6.37278 \mathrm{E}-5$ & $3.08407 \mathrm{E}-5$ & $1.43934 \mathrm{E}-5$ & $6.17426 \mathrm{E}-6$ & $2.20966 \mathrm{E}-6$ \\
& 1.05 & 1.10 & 1.22 & 1.48 & \\
$2^{-8}$ & $6.52702 \mathrm{E}-5$ & $3.23831 \mathrm{E}-5$ & $1.59358 \mathrm{E}-5$ & $7.71119 \mathrm{E}-6$ & $3.59865 \mathrm{E}-6$ \\
& 1.01 & 1.02 & 1.05 & 1.10 & \\
$2^{-10}$ & $6.56558 \mathrm{E}-5$ & $3.27687 \mathrm{E}-5$ & $1.63214 \mathrm{E}-5$ & $8.09677 \mathrm{E}-6$ & $3.98423 \mathrm{E}-6$ \\
& 1.00 & 1.01 & 1.01 & 1.02 & \\
$2^{-12}$ & $6.57522 \mathrm{E}-5$ & $3.28651 \mathrm{E}-5$ & $1.64178 \mathrm{E}-5$ & $8.19317 \mathrm{E}-6$ & $4.08062 \mathrm{E}-6$ \\
& 1.00 & 1.00 & 1.00 & 1.01 & \\
$2^{-14}$ & $6.57763 \mathrm{E}-5$ & $3.28892 \mathrm{E}-5$ & $1.64419 \mathrm{E}-5$ & $8.21727 \mathrm{E}-6$ & $4.10472 \mathrm{E}-6$ \\
& 1.00 & 1.00 & 1.00 & 1.00 & \\
$2^{-16}$ & $6.57820 \mathrm{E}-5$ & $3.28949 \mathrm{E}-5$ & $1.64475 \mathrm{E}-5$ & $8.22291 \mathrm{E}-6$ & $4.11037 \mathrm{E}-6$ \\
& 1.00 & 1.00 & 1.00 & 1.00 & \\
$2^{-18}$ & $6.57837 \mathrm{E}-5$ & $3.28967 \mathrm{E}-5$ & $1.64493 \mathrm{E}-5$ & $8.22470 \mathrm{E}-6$ & $4.11216 \mathrm{E}-6$ \\
& 1.00 & 1.00 & 1.00 & 1.00 & \\
$2^{-20}$ & $6.57842 \mathrm{E}-5$ & $3.28971 \mathrm{E}-5$ & $1.64498 \mathrm{E}-5$ & $8.22515 \mathrm{E}-6$ & $4.11260 \mathrm{E}-6$ \\
& 1.00 & 1.00 & 1.00 & 1.00 & \\
$e^{N}$ & $6.57842 \mathrm{E}-5$ & $3.28971 \mathrm{E}-5$ & $1.64498 \mathrm{E}-5$ & $8.22515 \mathrm{E}-6$ & $4.11260 \mathrm{E}-6$ \\
$p^{N}$ & 1.00 & 1.00 & 1.00 & 1.00 & \\
\hline
\end{tabular}

Now, we define the exact error $e_{\varepsilon}^{N}$ and the computed parameter-uniform maximum pointwise error $e^{N}$ as follows:

$$
e_{\varepsilon}^{N}=\|y-u\|_{\infty, \bar{\omega}}, e^{N}=\max _{\varepsilon} e_{\varepsilon}^{N},
$$


where $y$ is the numerical approximation to $u$ for various values of $N, \varepsilon$. We also define the computed parameter-uniform rate of convergence to be

$$
p^{N}=\log _{2}\left(e^{N} / e^{2 N}\right) .
$$

The values of $\varepsilon$ and $N_{0}\left(N=3 N_{0} / 2\right)$ for which we solve the test problems are $\varepsilon=2^{-2 i}, i=1,2, \ldots, 10 ; N_{0}=64,128,256,512,1024$. The resulting errors $e^{N}$ and the corresponding numbers $p^{N}$ after 20 iterations are given in Tables 1-3.

TABLE 2. Errors and convergence rates for Example 2

\begin{tabular}{llllll}
\hline$\varepsilon$ & $N_{0}=64$ & $N_{0}=128$ & $N_{0}=256$ & $N_{0}=512$ & $N_{0}=1024$ \\
\hline $2^{-2}$ & $2.36474 \mathrm{E}-5$ & $6.88645 \mathrm{E}-6$ & $1.8032 \mathrm{E}-6$ & $4.56462 \mathrm{E}-7$ & $1.34503 \mathrm{E}-7$ \\
& 1.78 & 1.93 & 1.98 & 1.76 & \\
$2^{-4}$ & $3.85540 \mathrm{E}-5$ & $1.65391 \mathrm{E}-5$ & $5.91880 \mathrm{E}-6$ & $1.72400 \mathrm{E}-6$ & $4.51464 \mathrm{E}-7$ \\
& 1.22 & 1.48 & 1.78 & 1.93 & \\
$2^{-6}$ & $4.26883 \mathrm{E}-5$ & $2.06587 \mathrm{E}-5$ & $9.64138 \mathrm{E}-6$ & $4.13580 \mathrm{E}-6$ & $1.48013 \mathrm{E}-6$ \\
& 1.05 & 1.10 & 1.22 & 1.48 & \\
$2^{-8}$ & $4.37216 \mathrm{E}-5$ & $2.16919 \mathrm{E}-5$ & $1.06746 \mathrm{E}-5$ & $5.16533 \mathrm{E}-6$ & $2.41053 \mathrm{E}-6$ \\
& 1.01 & 1.02 & 1.05 & 1.10 & \\
$2^{-10}$ & $4.39799 \mathrm{E}-5$ & $2.19502 \mathrm{E}-5$ & $1.09329 \mathrm{E}-5$ & $5.42362 \mathrm{E}-6$ & $2.66881 \mathrm{E}-6$ \\
& 1.00 & 1.01 & 1.01 & 1.02 & \\
$2^{-12}$ & $4.40444 \mathrm{E}-5$ & $2.20148 \mathrm{E}-5$ & $1.09974 \mathrm{E}-5$ & $5.48819 \mathrm{E}-6$ & $2.73338 \mathrm{E}-6$ \\
& 1.00 & 1.00 & 1.00 & 1.01 & \\
$2^{-14}$ & $4.40606 \mathrm{E}-5$ & $2.20309 \mathrm{E}-5$ & $1.10136 \mathrm{E}-5$ & $5.50433 \mathrm{E}-6$ & $2.74952 \mathrm{E}-6$ \\
& 1.00 & 1.00 & 1.00 & 1.00 & \\
$2^{-16}$ & $4.40643 \mathrm{E}-5$ & $2.20346 \mathrm{E}-5$ & $1.10173 \mathrm{E}-5$ & $5.50805 \mathrm{E}-6$ & $2.75325 \mathrm{E}-6$ \\
& 1.00 & 1.00 & 1.00 & 1.00 & \\
$2^{-18}$ & $4.40655 \mathrm{E}-5$ & $2.20359 \mathrm{E}-5$ & $1.10185 \mathrm{E}-5$ & $5.50930 \mathrm{E}-6$ & $2.75449 \mathrm{E}-6$ \\
& 1.00 & 1.00 & 1.00 & 1.00 & \\
$2^{-20}$ & $4.40659 \mathrm{E}-5$ & $2.20362 \mathrm{E}-5$ & $1.10189 \mathrm{E}-5$ & $5.50961 \mathrm{E}-6$ & $2.75480 \mathrm{E}-6$ \\
& 1.00 & 1.00 & 1.00 & 1.00 & \\
$e^{N}$ & $4.40659 \mathrm{E}-5$ & $2.20362 \mathrm{E}-5$ & $1.10189 \mathrm{E}-5$ & $5.50961 \mathrm{E}-6$ & $2.75480 \mathrm{E}-6$ \\
$p^{N}$ & 1.00 & 1.00 & 1.00 & 1.00 & \\
\hline
\end{tabular}

\section{CONCLUSion}

In this paper, we have developed a finite difference method for solving the singularly perturbed nonlocal boundary-value problem for a second order delay differential equation. This method was based on an exponentially fitted difference scheme on a uniform mesh. The method was proved to be first order accuracy in the discrete maximum norm, with respect to the perturbation parameter $\varepsilon$. Test problems were 
TABLE 3. Errors and convergence rates for Example 3

\begin{tabular}{llllll}
\hline$\varepsilon$ & $N_{0}=64$ & $N_{0}=128$ & $N_{0}=256$ & $N_{0}=512$ & $N_{0}=1024$ \\
\hline $2^{-2}$ & $3.67457 \mathrm{E}-5$ & $1.07011 \mathrm{E}-5$ & $2.80203 \mathrm{E}-6$ & $8.90948 \mathrm{E}-7$ & $3.19656 \mathrm{E}-7$ \\
& 1.78 & 1.94 & 1.65 & 1.48 & \\
$2^{-4}$ & $5.99030 \mathrm{E}-5$ & $2.57004 \mathrm{E}-5$ & $9.19987 \mathrm{E}-6$ & $2.68102 \mathrm{E}-6$ & $7.89100 \mathrm{E}-7$ \\
& 1.22 & 1.48 & 1.78 & 1.93 & \\
$2^{-6}$ & $6.63273 \mathrm{E}-5$ & $3.21016 \mathrm{E}-5$ & $1.49846 \mathrm{E}-5$ & $6.43084 \mathrm{E}-6$ & $2.30438 \mathrm{E}-6$ \\
& 1.05 & 1.10 & 1.22 & 1.48 & \\
$2^{-8}$ & $6.79328 \mathrm{E}-5$ & $3.37071 \mathrm{E}-5$ & $1.65901 \mathrm{E}-5$ & $8.03061 \mathrm{E}-6$ & $3.75060 \mathrm{E}-6$ \\
& 1.01 & 1.02 & 1.05 & 1.10 & \\
$2^{-10}$ & $6.83341 \mathrm{E}-5$ & $3.41084 \mathrm{E}-5$ & $1.69915 \mathrm{E}-5$ & $8.43195 \mathrm{E}-6$ & $4.15194 \mathrm{E}-6$ \\
& 1.00 & 1.01 & 1.01 & 1.02 & \\
$2^{-12}$ & $6.84344 \mathrm{E}-5$ & $3.42087 \mathrm{E}-5$ & $1.70918 \mathrm{E}-5$ & $8.53229 \mathrm{E}-6$ & $4.25228 \mathrm{E}-6$ \\
& 1.00 & 1.00 & 1.00 & 1.01 & \\
$2^{-14}$ & $6.84595 \mathrm{E}-5$ & $3.42338 \mathrm{E}-5$ & $1.71169 \mathrm{E}-5$ & $8.55737 \mathrm{E}-6$ & $4.27736 \mathrm{E}-6$ \\
& 1.00 & 1.00 & 1.00 & 1.00 & \\
$2^{-16}$ & $6.84656 \mathrm{E}-5$ & $3.42397 \mathrm{E}-5$ & $1.71227 \mathrm{E}-5$ & $8.56318 \mathrm{E}-6$ & $4.28313 \mathrm{E}-6$ \\
& 1.00 & 1.00 & 1.00 & 1.00 & \\
$2^{-18}$ & $6.84673 \mathrm{E}-5$ & $3.42416 \mathrm{E}-5$ & $1.71246 \mathrm{E}-5$ & $8.56511 \mathrm{E}-6$ & $4.28510 \mathrm{E}-6$ \\
& 1.00 & 1.00 & 1.00 & 1.00 & \\
$2^{-20}$ & $6.84677 \mathrm{E}-5$ & $3.42420 \mathrm{E}-5$ & $1.71251 \mathrm{E}-5$ & $8.56558 \mathrm{E}-6$ & $4.28557 \mathrm{E}-6$ \\
& 1.00 & 1.00 & 1.00 & 1.00 & \\
$e^{N}$ & $6.84677 \mathrm{E}-5$ & $3.42420 \mathrm{E}-5$ & $1.71251 \mathrm{E}-5$ & $8.56558 \mathrm{E}-6$ & $4.28557 \mathrm{E}-6$ \\
$p^{N}$ & 1.00 & 1.00 & 1.00 & 1.00 & \\
\hline
\end{tabular}

addressed for different cases of $l_{1}$ and $r$. The case of $l_{1}=r$ was obtained better results than in other cases. Furthermore, the approximate errors and the rates of convergence for test problems was computed for different values of $\varepsilon$ and $N_{0}$ in Tables 1-3. Numerical results were carried out to show the efficiency and accuracy of the method. Theoretical results represent undergoing more complicated delay problems.

\section{REFERENCES}

[1] N. Adžić, "Spectral approximation and nonlocal boundary value problem." Novi. Sad. J. Math., vol. 30, no. 3, pp. 1-10, 2000.

[2] G. M. Amiraliyev and M. Cakir, "Numerical solution of the singularly perturbed problem with nonlocal boundary condition." Appl. Math. Mech. Eng. Edt., vol. 23, no. 7, pp. 755-764, 2002, doi: 10.1007/BF02456971.

[3] G. M. Amiraliyev and E. Cimen, "Numerical method for a singularly perturbed convectiondiffusion problem with delay." Appl. Math. Comput., vol. 216, no. 8, pp. 2351-2359, 2010, doi: 10.1016/j.amc.2010.03.080. 
[4] V. B. Andreev, "The Green function and a priori estimates of solutions of monotone three-point singularly perturbed finite-difference schemes." Diff. Equat., vol. 37, no. 7, pp. 923-933, 2001, doi: 10.1023/A:1011949419389.

[5] C. Bai and X. Xu, "Positive solutions for a functional delay second-order three-point boundaryvalue problem.” Electron. J. Diff. Equat., vol. 2006, no. 41, pp. 1-10, 2006.

[6] M. Cakir and G. M. Amiraliyev, "Numerical solution of a singularly perturbed three-point boundary value problem.” Int. J. Comput. Math., vol. 84, no. 10, pp. 1465-1481, 2007, doi: 10.1080/00207160701296462.

[7] J. Chen, J. Tariboon, and S. Koonprasert, "Existence of positive solutions to a second-order multipoint boundary value problem with delay." Thai J. Math., vol. 8, no. 4, pp. 21-32, 2010.

[8] E. Cimen, "A priori estimates for solution of singularly perturbed boundary value problem with delay in convection term." J. Math. Anal., vol. 8, no. 1, pp. 202-211, 2017.

[9] M. W. Derstein, H. M. Gibbs, F. A. Hopf, and D. L. Kaplan, "Bifurcation gap in a hybrid optically bistable system." Phys. Rev. A, vol. 26, no. 6, pp. 3720-3722, 1982, doi: 10.1103/PhysRevA.26.3720.

[10] E. Els'gol'ts, Qualitative Methods in Mathematical Analysis, in: Translations of Mathematical Monographs, vol.12. Providence, RI: AMS, 1964.

[11] E. Erneux, Applied Delay Differential Equations. New York: Springer, 2009.

[12] F. Z. Geng and S. P. Qian, "Modified reproducing kernel method for singularly perturbed boundary value problems with a delay." Appl. Math. Model., vol. 39, no. 18, pp. 5592-5597, 2015, doi: 10.1016/j.apm.2015.01.021.

[13] V. Y. Glizer, "Controllability conditions of linear singularly perturbed systems with small state and input delays." Math. Control Signals Syst., vol. 28, no. 1, pp. 1-29, 2016, doi: 10.1007/s00498015-0152-3.

[14] V. A. Il'in and E. I. Moiseev, "Nonlocal boundary-value problem of the first kind for a SturmLiouville operator in its differential and finite difference aspects." Diff. Equat., vol. 23, no. 7, pp. 803-810, 1987.

[15] T. Jankowski, "Solvability of three point boundary value problems for second order differential equations with deviating arguments." J. Math. Anal. Appl., vol. 312, no. 2, pp. 620-636, 2005, doi: 10.1016/j.jmaa.2005.03.076.

[16] C. G. Lange and R. M. Miura, "Singular perturbation analysis of boundary value problems for differential-difference equations." SIAM J. Appl. Math., vol. 42, no. 3, pp. 502-531, 1982, doi: $10.1137 / 0142036$.

[17] L. B. Liu and Y. Chen, "Maximum norm a posteriori error estimates for a singularly perturbed differential difference equation with small delay." Appl. Math. Comput., vol. 227, no. 15, pp. 801-810, 2014, doi: 10.1016/j.amc.2013.10.085.

[18] A. Longtin and J. Milton, "Complex oscillations in the human pupil light reflex with mixed and delayed feedback." Math. Biosci., vol. 90, no. 1, pp. 183-199, 1988, doi: 10.1016/00255564(88)90064-8.

[19] R. Ma, “A survey on nonlocal boundary value problems.” Appl. Math. E-Notes, vol. 7, pp. 257279, 2007.

[20] M. C. Mackey and L. Glass, "Oscillations and chaos in physiological control systems." Science, vol. 197, no. 4300, pp. 287-289, 1977, doi: 10.1126/science.267326.

[21] H. G. Roos, M. Stynes, and L. Tobiska, Robust Numerical Methods for Singularly Perturbed Differential Equations. Berlin: Springer-Verlag, 2008.

[22] R. B. Stein, "Some models of neuronal variability." Biophys J., vol. 7, no. 1, pp. 37-68, 1967, doi: 10.1016/S0006-3495(67)86574-3. 
[23] V. Subburayan and N. Ramanujam, "Asymptotic initial value technique for singularly perturbed convection-diffusion delay problems with boundary and weak interior layers." Appl. Math. Lett., vol. 25, no. 12, pp. 2272-2278, 2012, doi: 10.1016/j.aml.2012.06.016.

[24] W. Wang and J. Shen, "Positive solutions to a multi-point boundary value problem with delay." Appl. Math. Comput., vol. 188, no. 1, pp. 96-102, 2007, doi: 10.1016/j.amc.2006.09.093.

[25] H. Zarin, "On discontinuous Galerkin finite element method for singularly perturbed delay differential equations." Appl. Math. Comput., vol. 38, no. 1, pp. 27-32, 2014, doi: 10.1016/j.aml.2014.06.013.

Authors' addresses

\section{Erkan Cimen}

Department of Mathematics, Faculty of Education, Van Yuzuncu Yil University, 65080 Van, Turkey

E-mail address: cimenerkan@hotmail.com

\section{Musa Cakir}

Department of Mathematics, Faculty of Science, Van Yuzuncu Yil University, 65080 Van, Turkey

E-mail address: cakirmusa@hotmail.com 